\title{
MEAN PLATELET VOLUME ON SYSTEMIC LUPUS ERYTHEMATOSUS PATIENTS WITH AND WITHOUT THROMBOCYTOPENIA IN DR HASAN SADIKIN HOSPITAL BANDUNG : A DESCRIPTIVE STUDY
}

\author{
Patrick Philo1, Dewi Kartika Turbawaty², Laniyati Hamijoyo ${ }^{3}$
}

${ }^{1}$ Faculty of Medicine Universitas Padjadjaran, Bandung, Indonesia

${ }^{2}$ Department of Clinical Pathology, Faculty of Medicine Universitas Padjadjaran/Dr. Hasan Sadikin Hospital, Bandung, Indonesia

${ }^{3}$ Department of Internal Medicine, Rheumatology Division, Faculty of Medicine Universitas Padjadjaran/Dr. Hasan Sadikin Hospital, Bandung, Indonesia

A R T I C L E I N F O

\section{Keywords:}

Mean Platelet Volume

Thrombocytopenia

Systemic Lupus Erythematosus

Platelet count

\section{Corresponding author:}

Laniyati Hamijoyo

E-mail address:

hamijoyo@yahoo.com

All authors have reviewed and approved the final version of the manuscript.

https://doi.org/10.37275/IJR.v10i1.1

\begin{abstract}
A B S T R A C T
Background : The aim of the study was to provide an insight about mean platelet volume (MPV) value in systemic lupus erythematosus (SLE) with and without thrombocytopenia patient. MPV was expected to be used to determine the cause thrombocytopenia in lupus, so the patient could be treated appropriately. Method : The study design was descriptive categoric, and the data were obtained by using crosssectional method from patient's medical record and lab examination result in the period from January $1^{\text {st }} 2016$ - January $31^{\text {st }} 2018$. The sampling method are done using total sampling. The inclusion criteria of this study were SLE patients diagnosed with SLE ACR (American College of Rheumatology) Criteria 1997, which MPV and platelet count had been examined at the same time, the data used is data that was first discovered in the period of the study. The exclusion criteria were incomplete medical record data (the data needed for patient's characteristic wasn't recorded), patient with thrombocytosis, and SLE with comorbidity such as thrombotic disease (ischemic stroke and deep vein thrombosis), other high inflammatory overlap diseases (such as rheumatoid arthritis and inflammatory bowel disease), and infection. Result : From 75 patients that match with the inclusion criteria, all patients were female and based on the age of diagnosis, most patients were in age group of 25-34 years old $(41,33 \%)$. Based on the lab results, group with normal platelet count have $53(70,7 \%)$ subjects of normal MPV and 12 (16\%) subjects of high MPV, while group with thrombocytopenia have $6(8 \%)$ subjects of normal MPV and $4(5,3 \%)$ subjects of high MPV. Conclusion : Group with normal MPV value and normal platelet count has the largest proportion, while the group with thrombocytopenia in lupus and high MPV value has the lowest proportion.
\end{abstract}

\section{Introduction}

Systemic Lupus Erythematosus (SLE) is an autoimmune disease with a wide spectrum of clinical manifestations and involving the role of autoantibodies. Tissue injury is mainly mediated by deposition of immune complex and binding of antibodies to a wide variation of body tissue. This disease is characterized by its chronic nature and has remitting and relapsing phase. ${ }^{1,2}$
Lupus has broad spectrum of clinical manifestations such as skin and mucosal, joint, blood, lungs, heart, renal, nervous system, and immune system involvement. ${ }^{3}$ Thrombocytopenia, which generally defined by platelet count below $150.000 / \mathrm{mm}^{3}$, is one of the haematological manifestation that commonly occurs in SLE patient. ${ }^{4,5}$ The low platelet count may affect thrombocyte volume 
in an inverse relationship to maintain constant thrombocyte mass. ${ }^{6}$ Thrombocytopenia in lupus may appear because of thrombocyte destruction mediated by antiplatelet antibodies, on the other hand, thrombocytopenia may also occur as the side effect of immunosuppressant medication. It is difficult to determine whether the occurence of thrombocytopenia in lupus arise due to disease activity or as a side effect of medication. 7,8

The average thrombocyte volume in the circulation can be measured with Mean Platelet Volume (MPV) examination, with the normal range of 7.5-11.5 fL. ${ }^{9}$ MPV value in SLE patient commonly associated with its inflammation degree, while in thrombocytopenia, MPV value may be used to differentiate its etiology (hyperdestructive or hypoproductive). ${ }^{6}, 10$

Previous study had shown the role of MPV in differentiating the etiology of thrombocytopenia. 10 Therefore, it might be used to identify the cause of thrombocytopenia in lupus. However, the study about MPV value on thrombocytopenia in lupus patient haven't been done, so there isn't any valid data yet. Besides, the study about MPV value on SLE patient has only been conducted abroad, hence there isn't any proven data in Indonesia so far. The aim of the study is to provide an insight about MPV value in SLE patient with and without thrombocytopenia. Hopefully, MPV was expected to be used to determine the cause of thrombocytopenia in lupus in the future, so the patient could be treated appropriately.

\section{Research Methods}

The study design was descriptive categoric, and the data were obtained by using cross-sectional method. The study was conducted in June-August 2018 using secondary data of the inpatient and outpatient medical record in the medical record installation, result of the laboratory examination in clinical pathology laboratorium, and Lupus Registry in Immunology Study Centre in Dr. Hasan Sadikin Hospital Bandung from January 2016-2018. The population of this study were SLE patient registered at lupus registry in immunology study centre and at rheumatology division of internal medicine department Dr. Hasan Sadikin Hospital Bandung in the period of January $1^{\text {st }} 2016$ January $31^{\text {st }}$ 2018. Data collection technique in this study was total sampling method. The inclusion criteria of this study were SLE patients diagnosed with SLE ACR (American College of Rheumatology) Criteria 1997, which MPV and platelet count had been examined at the same time, the data used is data that was first discovered in the period of the study. The exclusion criteria were incomplete medical record data (the data needed for patient's characteristic wasn't recorded), patient with thrombocytosis, and SLE with comorbidity such as thrombotic disease (ischemic stroke and deep vein thrombosis), other high inflammatory overlap diseases (such as rheumatoid arthritis and inflammatory bowel disease), and infection.

MPV and platelet count were measured using Sysmex $X N-1000$ with the flow cytometry principle. The reference value used in the study was based on the Clinical Pathology Laboratory at Dr. Hasan Sadikin Hospital Bandung with value of 7.2-11.1 fL and $150.000-450.000 / \mathrm{mm}^{3}$ respectively for MPV and platelet count.

This study had been approved by the Research Ethical Committee of Universitas Padjadjaran, Bandung through the letter of approval Number 183/UN6.KEP/EC/2018. Data were collected from the patient's MPV and platelet count result, then the data was categorized based on the MPV (low, normal, high) and platelet count (normal and thrombocytopenia) interpretation. Demographical data were obtained from medical record and lupus registry. Data analyzed using descriptive statistic, processed using Microsoft Excel 2016 and presented in tables and percentage.

\section{Results}

During the period between January $1^{\text {st }}$ 2016January $31^{\text {st }} 2018,104$ SLE patients with 114 laboratory examination results were collected and fulfilled the inclusion criteria of this study. As many as 39 data among the 114 lab result were met the exclusion criteria, with 27 data were excluded due to infection, 12 data excluded due to thrombosis disease, 
thrombocytosis, and incomplete medical record data. Therefore, there were 75 SLE patients and lab examination results which included in this study.

The study show that all subject of this study (100\%) are female, and most subject are diagnosed with SLE at the age group of 25-34 years old (41.3\%), followed by age group 15-24 years old (37.4\%). Based on the duration of patient diagnosed with SLE until laboratory examination are conducted, most subject are having laboratory examination in 0-2 years (57.3\%) and 3-4 years after diagnosed with SLE (16\%).
Among 75 lab examination data, normal MPV value are found in 59 subjects $(78.7 \%)$ and the other 16 subjects $(21.3 \%)$ have a high MPV value, while normal platelet count are found in 65 subjects (86.7\%) and 10 subjects (13.3\%) have thrombocytopenia. Among 65 subjects with normal platelet count, 53 subjects $(70.7 \%)$ have normal MPV value and 12 subjects $(16 \%)$ have high MPV value, whereas the other 10 subjects with thrombocytopenia, normal MPV value are found in 6 subjects $(8 \%)$ and high MPV value are found in 4 subjects $(5.3 \%)$

Table 1. Characteristics of study's subject

\begin{tabular}{lcc}
\hline Variable & Subject (n=75) & Percentage (\%) \\
\hline $\begin{array}{l}\text { Gender } \\
\text { - Female }\end{array}$ & 75 & 100 \\
Age at diagnosis & & \\
- $\quad 15$ years old & 3 & 4 \\
- 15-24 years old & 28 & 37.4 \\
- 25-34 years old & 31 & 41.3 \\
- 35-44 years old & 10 & 13.3 \\
- 45-54 years old & 3 & 4 \\
\hline
\end{tabular}

\begin{tabular}{lcc}
\hline Variable Subject (n=75) & Percentage (\%) \\
\hline & & \\
Duration of patient diagnosed & & \\
with SLE until MPV and platelet & & \\
count examination are & & \\
conducted & 43 & 57.3 \\
- 0-2 years & 12 & 16 \\
- 3-4 years & 5 & 6.7 \\
- 5-6 years & 6 & 8 \\
- 7-8 years & 9 & 12 \\
- 8 years & & \\
\hline
\end{tabular}

Table 2. Mean Platelet Volume Value of SLE Patient in Hasan Sadikin Hospital between January 1 st 2016 January 31 st 2018

\begin{tabular}{lll}
\hline Mean Platelet Volume & $\mathbf{N}=\mathbf{7 5}$ & $\%$ \\
\hline Normal & 59 & $78.7 \%$ \\
High & 16 & $21.3 \%$ \\
\hline Total & 75 & $100 \%$ \\
\hline
\end{tabular}

*Reference Value : Normal (7.2-11.1 fL). High (>11.1 fL) 
Table 3. Platelet Count Value of SLE Patient in Hasan Sadikin Hospital between January 1 st 2016 - January 31 st 2018

\begin{tabular}{lll}
\hline Platelet Count & $\mathbf{N}=\mathbf{7 5}$ & $\%$ \\
\hline Normal & 65 & $86.7 \%$ \\
Thrombocytopenia & 10 & $13.3 \%$ \\
\hline Total & 75 & $100 \%$
\end{tabular}

*Reference Value : Normal (150.000-450.000/ $\left.\mathrm{mm}^{3}\right)$. Thrombocytopenia $\left(<150.000 / \mathrm{mm}^{3}\right)$

Table 4. Mean Platelet Volume Value of SLE Patient with \& without thrombocytopenia in Hasan Sadikin Hospital between January 1 st 2016 - January 31 st 2018

\begin{tabular}{lllll} 
& & \multicolumn{2}{l}{} & \\
\cline { 3 - 5 } & & Platelet Count (n= 75) & Total \\
\cline { 2 - 5 } $\begin{array}{l}\text { Mean Platelet } \\
\text { Volume } \\
(\mathbf{n = 7 5 )}\end{array}$ & Normal & $6(8 \%)$ & $53(70.7 \%)$ & $59(78.7 \%)$ \\
\cline { 2 - 5 } & High & $4(5.3 \%)$ & $12(16 \%)$ & $16(21.3 \%)$ \\
\hline & Total & $10(13.3 \%)$ & $65(86.7 \%)$ & $75(100 \%)$ \\
\hline
\end{tabular}

\section{DISCUSSION}

From this study, it was found that all subjects of this study $(100 \%)$ were female and 31 patient $(41.3 \%)$ among them were diagnosed at the age of 25-34 years old. This result is similar with the study from Rupert W. Jakes et al in South-East Asia (2012) who reported that $83-97 \%$ of SLE patient in South-East Asia are female, with the mean age of $25.7-35.4$ years old when diagnosed. ${ }^{11}$ Another study conducted by F Ress et al (2017) also stated that the prevalence of SLE is higher in woman at reproductive age than man with the ratio of $1.2-15: 1$ globally. 12

Hormone regulation and its effect to cytokine production, along with chromosomal factor, play a central role in causing the high proportion of SLE in woman at reproductive age. Estrogen and Prolactin are capable to stimulate autoreactive B Lymphocyte cell, hence leading to loss of immune tolerance and secretion of autoantibody. ${ }^{13}$ On the other hand, Androgen and Testosterone have protective nature to autoimmune disease, because both are natural immunosuppressant. ${ }^{14}$

Haematological abnormality (thrombocytopenia, anemia, leucopenia) is a common manifestation of SLE, it may appear on the onset disease or develop along the disease course, as the consequence of disease activity or induced by drugs. 8,15 In this study, "duration of patient diagnosed with SLE until laboratory examination are conducted" refer to duration of patient diagnosed with SLE until the occurence of haematological manifestation, because MPV and platelet count only done on SLE patient with haematological abnormality in Hasan Sadikin hospital. This study showed that most subject are having MPV and platelet count examination conducted in the first 2 years (57.3\%) after diagnosed. The result is equal with the study of Aamer Aleem et al in Arab Saudi (2014) who reported that $516(82.7 \%)$ out of 624 SLE patients are having haematological manifestation at the onset of diagnosis.16 High frequency of haematological manifestation in SLE patient is caused by many and varied antigens on blood cells and blood vessels. In autoimmune case, autoantibody will be produced against self-antigen, including blood cells, and haematological abnormality will appear through inflammation. 17, 18

MPV has an important role in high inflammatory disease like SLE and rheumatoid arthritis, it might be used as a marker of inflammation degree, a marker of disease activity, and to measure the efficacy of antiinflammatory therapy in some inflammatory disease. ${ }^{6}$ The MPV value of SLE patient without thrombocytopenia in this study were normal in 53 subjects $(70.7 \%)$ and high in 12 subjects (16\%). This result resembles the research done by Sun-Yi Chen et al in China (2018) who found 91 SLE patients have mean MPV value of $10.74 \pm 0.94 \mathrm{fL}$ (International Reference : 7.2-11.5 fL), which interpreted to normal- 
high. This result is lower than the control (mean MPV $=11.09 \pm 1.14 \mathrm{fL}$ ), which show the effect of inflammation on MPV value. 19

The role of MPV as the marker of inflammation degree and disease activity also illustrated by the study of Sahin Safak et al in Turkey (2014) who conclude that MPV value of group with active arthritis is lower than the group with inactive arthritis, while the control group have the highest value. ${ }^{20}$ Similar result also stated by the study conducted by Guillermo DelgadoGarcia et al in Mexico (2016), who showed that a group of patients with active SLE have a lower MPV value $(\mathrm{MPV}=7.16 \pm 1.39)$ than a group of SLE patients whose disease are at remission $(\mathrm{MPV}=8.16 \pm 1.50) .{ }^{21} \mathrm{In}$ high inflammatory disease like SLE, MPV has a low value because there is an increase in inflammatory cytokine production like IL-3 and IL-6, which will boost the thrombopoiesis (thrombocyte production). The process will result in large thrombocyte, but this large thrombocyte will be consumed at the site of inflammation, hence only small thrombocyte left in the circulation. 6, 19

The MPV value of thrombocytopenia in lupus patient this study showed normal in 6 subjects $(8 \%)$ and high in 4 subjects (5.3\%). This result unabled to be compared, because there was not any preliminary research about MPV value on thrombocytopenia in lupus patient. However, MPV can be used to distinguish thrombocytopenia based on its cause. MPV value tend to be higher on thrombocytopenia caused by excess platelet destruction (hyperdestructive thrombocytopenia) like immune thrombocytopenic purpura, disseminated intravascular coagulation, sepsis, and preeclampsia. While MPV value is prone to be lower on thrombocytopenia caused by reduced thrombocyte production (Hypoproductive Thrombocytopenia) like aplastic anemia. 9, 22

The difference in MPV value on thrombocytopenia based on its etiology was suitable with the result of the study conducted by Numbenjapon et al in Thailand (2008). Among 62 patients with hyperdestructive thrombocytopenia, 51 subjects have MPV value $>7.9$ $\mathrm{fL}$ and 11 other have MPV value $\leq 7.9 \mathrm{fL}$. From 40 patients with hypoproductive thrombocytopenia, 37 subjects have MPV value $\leq 7.9 \mathrm{fL}$, while the rest have MPV value $>7.9 \mathrm{fL}$. The result shows that group of hyperdestructive thrombocytopenia have a higher MPV value than group of hypoproductive thrombocytopenia. ${ }^{22}$ The distinction in MPV value may occur because in hyperdestructive case, excess platelet destruction will lead to compensation from bone marrow to boost the platelet production, so that large thrombocyte will be produced. 10

Thrombocytopenia is a common manifestation found in SLE patient and also one of the criteria for diagnosis of SLE. ${ }^{7}$ Thrombocytopenia usually appear through autoimmune mechanism similar to immune thrombocytopenic purpura and mediated by antiplatelet, but it may also emerge as a side effect of immunosuppressant therapy (methotrexate, cyclophosphamide, azathioprine, and hydroxychloroquine). ${ }^{8,23}$ However, it is still difficult to distinguish between thrombocytopenia in lupus caused by disease activity or side effect of therapy. ${ }^{7}$ Although there isn't any preliminary study about MPV value on thrombocytopenia in lupus patient, the prove that MPV can differentiate the cause of thrombocytopenia can be the first step in order to use MPV to distinguish the cause of thrombocytopenia in lupus patient, so that the patient can get appropriate treatment.

In this study, there was not any data with low MPV value. Theoretically, high disease activity is associated with high inflammatory degree that cause consumption of large thrombocyte and left small thrombocyte in circulation, which lead to lower MPV value. ${ }^{6}$ Study conducted by Sahin Safak et al in Turkey showed the MPV value of SLE patients with active arthritis are 7.66 \pm 0.89 (low - normal). ${ }^{20}$ Research done by Guillermo Delgado-García et al in Mexico also proved that patients with active SLE have a low MPV value (7.16 \pm 1.39 ), with the disease activity measured using Mex-SLEDAI (Mexican Systemic Lupus Erythematosus Disease Activity Index). Patient stated active if the Mex-SLEDAI score $>5$ and inactive if the Mex-SLEDAI socre < 2. 21 The differences in this study and the previous study might be caused by the limitation of this study, that in 
this study there were not any data about patient's disease activity, so that the laboratory result could not be adjusted with the disease activity. Besides, there are other factors that can interfere with the MPV value such as improper storage of blood samples, the use of antiinflammatory drugs, the probabilty of emergence of other comorbidity, presence of cardiovascular risk factor (smoking, hypertension, diabetes mellitus, obesity, and dyslipidemia), and the use of antihypertension.6,24 These factors are less noticed by author, so they could become limitations in this study, beside limited subject and incomplete data.

\section{CONCLUSION}

Lupus patient with both normal MPV value and normal platelet count has the largest proportion, while the group with thrombocytopenia and high MPV value has the lowest proportion.

Subsequent research could use primary data and focus on proving MPV value to distinguish the cause of thrombocytopenia in lupus, so that the patient can get appropriate therapy in the future.

\section{Acknowledgement}

The authors gratefully acknowledge the assistance of dr. Sasfia Candrianita in collecting patient's data from the Lupus Registry.

\section{REFERENCES}

1. Bertsias G, Cervera R, Boumpas DT. Systemic Lupus Erythematosus: Pathogenesis and Clinical Features. Eular On-line Course Rheum Dis. 2012;(1909):476-505.

2. Kumar V, Abbas AK, Aster JC. Diseases of the Immune System. In: Pathologic Basis of Disease. 9th ed. Philadelphia: Elsevier Saunders; 2015. p. 185-264.

3. Kasjmir YI, Handono K, Wijaya LK, Hamijoyo L, Albar Z, Kalim H, et al. Recommendation of Indonesian Rheumatology Association for Diagnosis and Treatment of Systemic Lupus Erythematosus [Rekomendasi Perhimpunan Reumatologi Indonesia untuk Diagnosis dan Pengelolaan Lupus Eritematosus Sistemik]. Indonesian Rheumatology Association [Perhimpunan Reumatologi Indonesia]; 2011. 1-
$54 \mathrm{p}$.

4. Jung J-H, Soh M-S, Ahn Y-H, Um Y-J, Jung J-Y, Suh C-H, et al. Thrombocytopenia in Systemic Lupus Erythematosus. Medicine (Baltimore). 2016;95(6): 1 .

5. Gauer RL, Braun MM. Thrombocytopenia. Am Fam Physician. 2012;85(6):613-22.

6. Gasparyan AY, Ayvazyan L, Mikhailidis DP, Kitas GD. Mean platelet volume: a link between thrombosis and inflammation? Curr Pharm Des. 2011;17(1):47-58.

7. Fayyaz A, Igoe A, Kurien BT, Danda D, James JA, Stafford HA, et al. Haematological manifestations of lupus. Lupus Sci Med. 2015;2(1):1-18.

8. Bashal F. Hematological Disorders in Patients with Systemic Lupus Erythematosus. Open Rheumatol J. 2013;7(1):87-95.

9. Schmoeller D, Picarelli MM, Paz Munhoz T, Poli de Figueiredo CE, Staub HL. Mean Platelet Volume and Immature Platelet Fraction in Autoimmune Disorders. Front Med. 2017;4(146):1-5.

10. Khairkar PS, More S, Pandey A, Pandey M. Role of mean platelet volume (MPV) in diagnosing categories of thrombocytopenia. Indian $\mathrm{J}$ Pathol Oncol. 2016;3(4):606.

11. Jakes RW, Bae S-C, Louthrenoo W, Mok C-C, Navarra S V., Kwon N. Systematic review of the epidemiology of systemic lupus erythematosus in the Asia-Pacific region: Prevalence, incidence, clinical features, and mortality. Arthritis Care Res (Hoboken). 2012;64(2):159-68.

12. Rees F, Doherty M, Grainge MJ, Lanyon P, Zhang W. The worldwide incidence and prevalence of systemic lupus erythematosus: a systematic review of epidemiological studies. Rheumatology. 2017;56:1945-61.

13. Schwartzman-Morris J, Putterman C. Gender differences in the pathogenesis and outcome of lupus and of lupus nephritis. Clin Dev Immunol. 2012;2012:1-9.

14. Ortona E, Pierdominici M, Maselli A, Veroni C, Aloisi F, Shoenfeld Y. Sex-based differences in autoimmune diseases. Ann Ise Super Sanita. 2016;52(2):205-12.

15. Teke HU, Cansu DU, Korkmaz C. Detailed features of hematological involvement and medicationinduced cytopenia in systemic lupus erythematosus patients: single center results of 221 patients. Eur J Rheumatol. 2017;4(2):87-92.

16. Aleem A, Al Arfaj AS, Khalil N, Alarfaj $\mathrm{H}$. Haematological abnormalities in systemic lupus erythematosus. Acta Reumatol Port. 2014;39(3):236-41.

17. Sasidharan PK, Bindya M, Sajeeth Kumar KG. Hematological Manifestations of SLE at Initial Presentation: Is It Underestimated? ISRN Hematol. 2012;2012:1-5. 
18. Fietta P, Delsante G, Quaini F. Hematologic manifestations of connective autoimmune diseases. Clin Exp Rheumatol. 2009;27(1):14054.

19. Chen SY, Du J, Lu XN, Xu JH. Platelet distribution width as a novel indicator of disease activity in systemic lupus erythematosus. J Res Med Sci. 2018;1-7.

20. Safak S, Uslu AU, Serdal K, Turker T, Soner S, Lutfi A. Association between mean platelet volume levels and inflammation in SLE patients presented with arthritis. Afr Health Sci. 2014;14(4):919-24.

21. Delgado-García G, Galarza-Delgado DÁ, ColungaPedraza I, Borjas-Almaguer OD, Mandujano-Cruz I, Benavides-Salgado D, et al. Mean platelet volume is decreased in adults with active lupus disease. Rev Bras Reumatol. 2016;56(6):504-8.
22. Numbenjapon $T$, Mahapo $N$, Pornvipavee $R$, Sriswasdi C, Mongkonsritragoon W, Leelasiri A, et al. A prospective evaluation of normal mean platelet volume in discriminating hyperdestructive thrombocytopenia from hypoproductive thrombocytopenia. Int $\mathrm{J}$ Lab Hematol. 2008;30(5):408-14.

23. Galanopoulos N, Christoforidou A, Bezirgiannidou $Z$. Lupus thrombocytopenia: pathogenesis and therapeutic implications. Mediterr J Rheumatol. 2017;28(1):30-6.

24. Chu SG, Becker RC, Berger PB, Bhatt DL, Eikelboom JW, Konkle B, et al. Mean platelet volume as a predictor of cardiovascular risk: a systematic review and meta-analysis. J Thromb Haemost. 2010;8(1):148-56. 